\title{
Incremental Identification of Inflectional Types
}

\author{
Petra Barg and James Kilbury \\ Hcinrich-Heine-Universität Düsseldorf, \\ Seminar für Allgemeine Sprachwissenschaft, \\ Universitätsstr. 1, D-40225 Diisscldorf, Germany \\ E-mail:\{barg,kilbury\}@(uling.uni-duesseldorf.de
}

\begin{abstract}
We present, an approach to tho incremental accrual of lexical information for unknown words that is comstraint-based and compatible with standard mification-based grammars. Although tho techniques are language-independent and can be applied to all kinds of information, in this paper we concentrate on the domain of German nom inflection. We show how morphological information, especially inflectional class, is successfully acquired using a tyy obased HPSG-like analysis. Furthornore, we sketch an alternative strategy which makes use of finitestate tiransducers.
\end{abstract}

\section{Introduction}

Systems for matural language processinge must deal adequately with "unknown" words, i.e. lexemes that either have been nowly coined or elso have not been included in a particular lexicon (cf. Kilbury of al. (1994)). Rather than sinply regarding unknown words as noise, our system instead uses their context as a somec for the systematic acerual of lexical information that can then be ntilized.

Our approach differs in significant respects from those of other investigators. It is clesigned for unification-based grammar formalisms with typed feature stuctures as in HPSG and is not restricted to simple morphosyntactic features. In contrast to statistical approaches like that of (Bront, 1991), which often do not work incrementally and are intended for the application to large corpora, ours instead aims at a detailed grammatical analysis of individual sentences with a maximal use of their information. While systems like that of (Hahn et al., 1996) deal with the general acquisition of concepts, we are concorned exclusively with the acquisition of structural linguistic information.

Although we deal here with German noun inflection, in a framowork close to that of (Richemann, 1998) and (Koonig, 1999), the techniques ano language-independent and apply to other kinds of lexical information as well, as is shown in (Walther and Barg, 1998) with respect to valency information. 'Thus, in contrast to (Ehrlich and Rapaport, 1997), who enploy tailored algorithms for the accuisition of information about nouns and verbs, we introduce an approach that is completely general with respect to the kind of structural linguistic information acguired.

\section{German noun-inflection classes}

'There is a vast literature on German nom inflection represented in recent studies by (Cahill and Gardar, 1999), (Clahsen, 1999), and (Neef, 1998). Here we summarize only essential points and ignore highly irregular and archaic inflections (cf. figure 4 below).

German nouns bear gender (masculine, feminine, neuter) and are inflected for number (singular, plural) and case (nominiative, accusative, dative, genitive). With the exception of class NWN (e.g. mase Bauer 'farmer", with gen sg Bauern), all nonfominine noms build genitive singular with $-s$.

the "regular" (of. Clahsen (1999)) but "atypical" (cf. Wunderlich (1999)) 11ouns of class NA (c.g. Auto 'car') build their plural forms in -s. 'I'he plural forms of all other (i.e. "typical") classes must end in a so-called schwa syllable-e, -el, -er, or -en (i.e. phonetically an unstressed [0] followed by a sonorant from $\left.\left[\begin{array}{lll}1 & r & 11\end{array}\right]\right)$.

Strong nouns add -e for plural in class NS (e.en. Arm 'arm', pl Arme) and class NU (e.g. Arzt "physi(cian', pl $\ddot{A r z t e}$ ) if the stem itself does not already end in a schwa syllable (e.g. Kabel 'cable', pl $\mathrm{Ka}$ $b c l$ ). Class NU furthermore umlauts the stem (i.e. replaces $a, o, u, a u$ with $\ddot{a}, \ddot{o}, \ddot{u}, \ddot{a} u$, respectively), as does class NR (e.g. Mann 'man', pl Männer), which adds - er:

'The remaining classes (NM, NWN, and NWS) form their plural in $-n$ (e.g. Schraube 'screw', pl Schrouben). 'The nomominative singular stem in class NWN (c.g. Hase 'hare', gen sg Hasen) and class NWS (e.g. Glaube 'belief', gen sg Glaubens) is identical with the plural form, while NWN exceptionally adds no $-s$ in grenitive singular.

All classes except NA build dative plural by adding $-n$ to the plural form if it is not alroady present (e.g. Männer 'mon', dat pl Männern but Hasen 'hares', dat pl Hasen). 
Figure 1: hierarchy of inflectional schemata

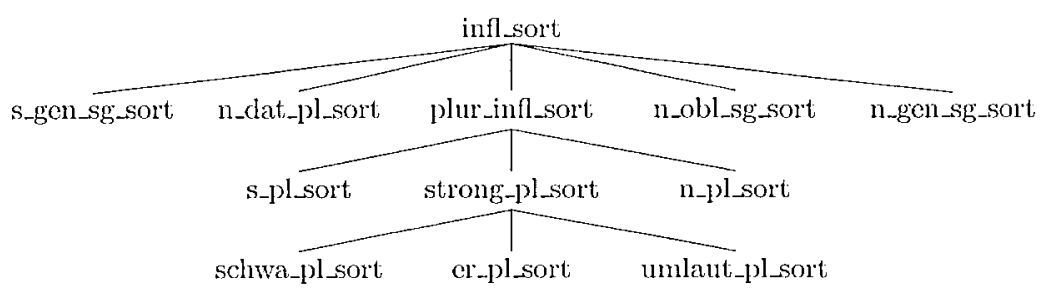

\section{Representation of inflectional morphology}

Various proposals have been made for the representation of inflectional morphology within constraintbased framoworks like HPSG (cf. Pollard (1994)). We neither adopt a word-syntax approach like that of (Krieger and Nerbonne, 1993) assuming lexical cntries for inflectional affixes as well as roots, nor do we make use of lexical rules, as (Mcurers and Minnen, 1997) do.

Instead, wo follow (Riehemann, 1998) in formulating hicrarchically structured schemata of the kind she has developed for derivational morphology but apply them here to inflection and thus carry out a kind of inflectional analysis without lexical rules as projected by (Erjavec, 1996). Our schemata capture inflectional paradigms and can be regarded as relational constraints that relate stems, affixes, and inflected lexical forms.

Figure 1 shows our hicrarchy of inflectional schemata, while figure 2 illustrates a concrete schema, namely that for the schwa plural of inflectional class NS. In figure 2 the attribute ftype stands for the inflectional class. The attributes flex, surf, and base represent strings, namely the inflectional ending, surface (i.e. inflected) form, and base form respectively. The symbol@denotes the reduced vowel [0] (schwa), and " designates negated values.

Lexical ontries are assumed only for basic lexical signs (i.c. uninflected but possibly derived or compounded). Inflected lexical signs result from the intcraction of these lexical cntrics and the inflectional schemata. Figure 3 gives the basic lexical sign (with the omission of feature specifications that are irrelevant for this discussion) for Hund 'dog', which is of class NS, followed by the inflected lexical sign for Hunde 'dogs', in which the value of the attribute moph (i.e. morphophonology) is an extension of the schema for schwa plural given in figure 2.

The inflectional classes assigned to basic lexical signs are modelled as formal types in the hicrarchical structure specified in figure 4 . Note that the leaves of this tree correspond exactly to the inflectional classes of German nouns as described above in $\S 2$.

Morphophonemic and morphographemic alterna-
Figure 2: schema for schwa-plural (schwa_pl_sort)

$$
\left[\begin{array}{ll}
\left.\begin{array}{ll}
\text { schwa_pl } \\
\text { ftype } & \text { ns } \\
\text { flex } & 0 \\
\text { surf } & \text { atom } \\
\text { base } & \text { atom } \\
\text { stem } & {\left[\begin{array}{l}
\text { basic_ls } \\
\text { moph basic }
\end{array}\right]} \\
\text { agr } & {\left[\begin{array}{l}
\text { agr } \\
\text { case }\left[\begin{array}{l}
\text { case_avm } \\
\text { ctxt } ~ \\
\text { gen topt }
\end{array}\right] \\
\text { num plu } \\
\text { gend } \sim f_{c m} \\
\text { pers }
\end{array}\right]}
\end{array}\right]
\end{array}\right]
$$

tions as shown in nominative plual Zeit-en 'times' but Gabet n 'forks' are also covered in our description. Here the realisation of the plural ending - $n$ depends on the shape of the noun stem (namely, whether or not; it ends in a schwa syllable). In agreoment with (Bird and Klein, 1994) and (Erjavec, 1996), we capture such alternations declaratively in a onc-level model without recourse to transducers. Our treatment of umlaut adopts part of the techniques of (Trost, 1993).

\section{Processing unknown words}

In our approach linguistic properties of unknown words are inferred from their sentential context as a byproduct of parsing. After parsing, which requires only a slight modification of standard loxical lookup, lexical entries are appropriately updated. One of our key ideas is a gradual, information-based concept of "unknownness", where lexical entrics arc not unknown as a whole, but may contain unknown, i.e. potentially revisable, pieces of information (cf. Barg and Walther (1998)). This allows a uniform treatment for the full range of lexical entries from completely known to maximally unknown. As discussed in (Barg and Walther, 1998), our system has been implemented in MicroCUF, a derivative of the formalism CUF of (Dörre and Dorna, 1993). 
Figure 3: foature structures for Hund and Hunde:

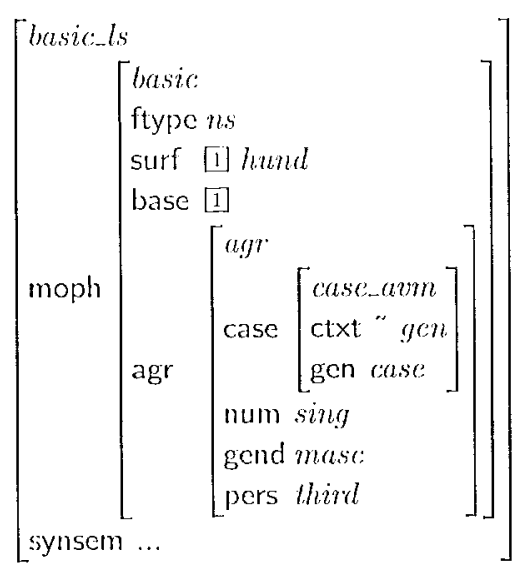

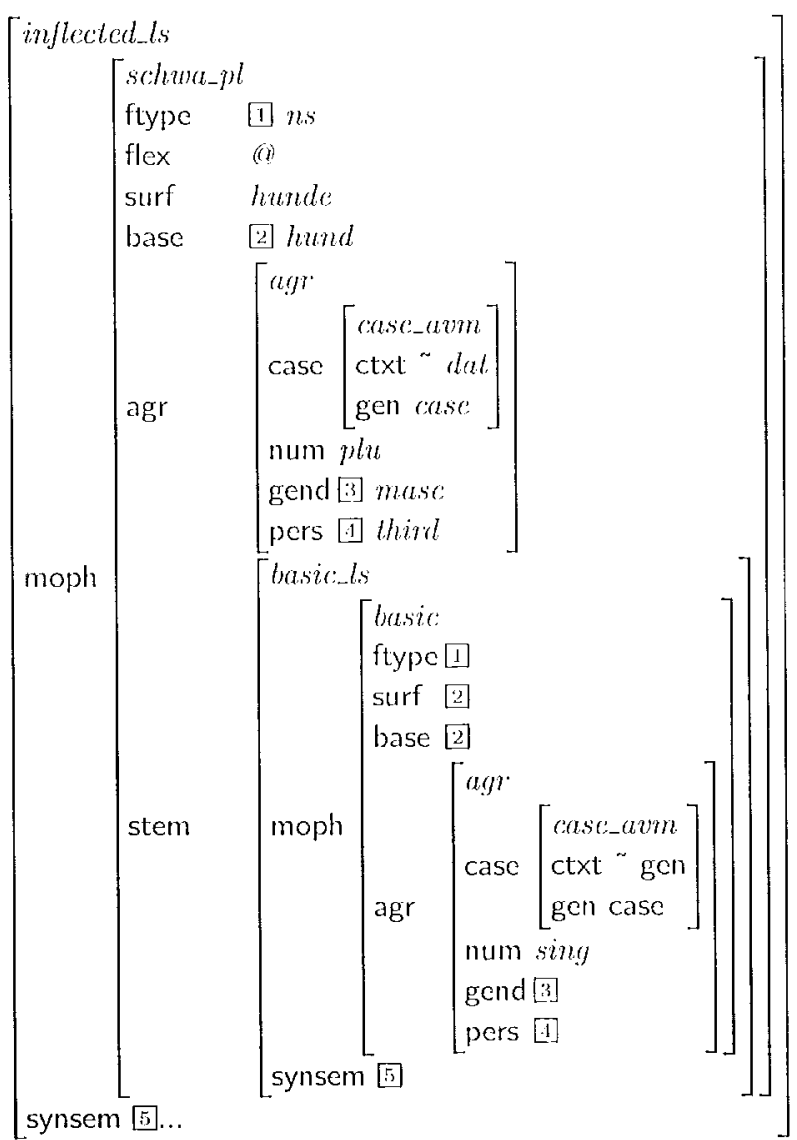

Revisable information is further classified as specializable or generalizable, where the former can only become more special, and the latter only more general, with further contexts. Specializable kinds of information include semantic type of nomms, gender, and inflectional class. Among the generalizible kinds of information are the selectional restrictions of verlos and adjectives as well as the case of nouns. IBoth kinds of information together with nonrevis- able (i.c. strict) information can cooccur in a single entiry.

']'he overall approach is compatible with standared constraint-based analyos and makes only a fow extra denands on the grammar. Here the revisable information must be explicitly marked as such. Sinco our model is situated within the framerork of typed feature-based formalisms (cf. Carpenter (1992)), revisable information is expressed in terms of formal typess. 'The initial values for' revisalble information ance specified with two distinguished types $u_{-} s$ and $u_{-} g$ for specializable and generalizable information, respectively. 'Lype unification can be employed for the combination of specializable information, whereas generalizable information requires type mutom.

The direct combination of revisable information doring parsing is unfeasible for various roasons discussed in (Barg and Walther, 1998). It conscquently is carried out, in a separate step) after the current sentence has been parsed. The grammatical analysis itself thus lemains completely declarative and only makes use of mification. In order to achieve this separation of analysis and revision we introduce two attributes for generalizalble information, namoly gen and ctxt, where ctxt receives the information infered from the sentential context, and gen the potentially revisable intormation with the initial value $u_{-} y$.

Parsing thens proceeds in an entirely convontional mamer, oxeept that lexical look-1up for a worel with unknown orthography or phonology does not fail but instead yjelds an underspecified canonical lexical entry. 'The updating' after parsing compares the foatino structure of the original lexical entry with that inferred contextually. Jhe specializable information of the former is replaced with the corresponding values of the lattier. Moreover, using the attributes gen and ctxt introduced above, the new gen value for generalizable information is computed by the type minom of the gen value from the old lexical ontry (initialy $u_{-} g$ ) with the ctxt value resulting from the parse. Actual revision naturally is only carried out when a context: in fact provicles new information.

\section{Incremental inference of inflectional information}

In order to process $11 n k$ known word forms, we postulate canonical lexical entries which are returned by lexical lookup if a word is not recorded in the lexicon. For nouns, this entry corresponds to an underspecificd basic lexical sign in which the inflectional class, case, number, and gender are specified with revisable types, i.e. the information can bo acquired and updated. Figure 5 shows the basic lexical sign for German nouns (with the omission of feature specifications that are irrelevant for this discussion).

Whereas inflectional class (ftype), number (num), 
Figure 4: hicrarchy of inflcctional types

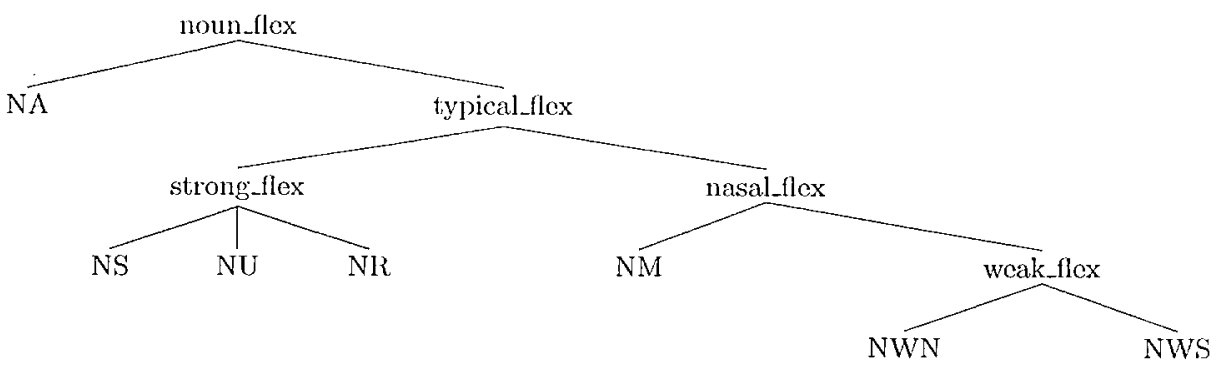

and gender (gend) are specializable, case is generalizable and hence contains the features gen and ctxt. Note that the initial values for specializable information consist of a disjunction $(;)$ of the value $u_{-} s$ and the most general appropriate value for the corresponding feature. This ensures the identification of specializable information (via $u_{-} s$ ) on the one hand, and the correct specializations on the other.

When a sentence containing an unknown noun is parsed, information about the noun comes from difforent sources: while the surrounding context may supply agrecment information, the word form itself together with morphophonological constraints may restrict the possible inflectional class.

As an example we can suppose that the rather infrequent noun Sund 'sound', 'strait', which like Hund 'dog' belongs to class NS but is unfamiliar to many German speakers, is not recorded in a given lexicon. The class NS contains both masculine and neuter nouns, and these differ in none of their inflected forms. Thus, only agreement information from a context, such as der enge Sund 'the narrow strait' (nominative), can establish the gender of Sund as being masculine.

Figure 5: feature structure for the underspecified lexical entry

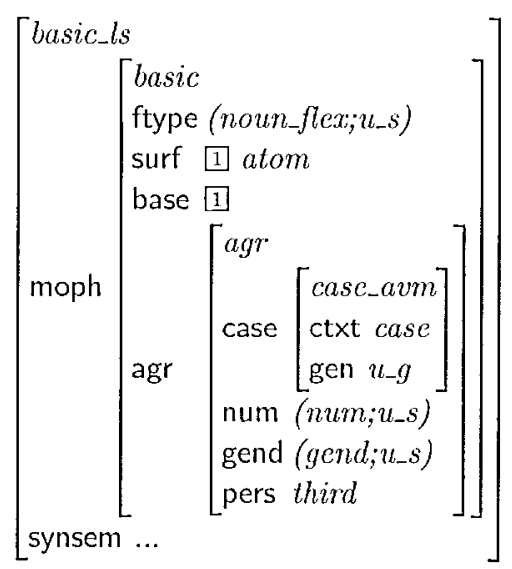

Even in isolation, the form Sund must be singu- lar since its final shape is not compatible with any plural inflection (i.e. it ends neither in $-s$ nor in a schwa syllable). Moreover, the morphophonological constraints on stems allow only threc possibilities: Sund is

- feminine (and then the class is NA, NU, or NM and the case is underspecified)

- nonfeminine and weak (i.c. class NWN or NWS) (and thon the case must be nominative)

- nonfeminine and nonweak (and then the case is not genitive)

These hypotheses are captured in the three feature structures depicted in figure 6 .

As we have seen, when a word is parsed in context, this provides additional information. If we know, for example, that Sund is masculine, the first hypothesis is excluded, and the gender specification of the remaining two hypotheses can be specializêd to masc. If we additionally encounter Sund in dative singular, which is impossible for woak nouns (which must have a final $-n$ ), then only the third hypothesis remains. Finally, if the plural form Sunde occurs the system can specialize the inflectional class exactly to the type NS. The other morphological information cannot be further generalized or specialized, and wo have the final lexical entry for Sund.

Things are not always this easy. In particular, there may be a number of alternatives both for the segmentation of a form into a stem and an inflectional ending and for the assignment of a stem to a lexeme. Morcover, these alternatives may depend on each other. Thus, the form Leinen may be assigned to any of the lexemes Lein 'flax' (masc, NS), Leine 'rope' (fem, NM), or Leinen 'linen' (neut, NS); even in a context, e.g. Fritz verkauft Leinen 'Fritz sells ropes/linen', it may be impossible to disambiguate the form. While the nouns Band 'book volume' (mase, NU), Band 'strip' (neut, NR), Band 'bond' (neut, NS, archaic and rare in singular), Band'music band' (fcm, NA), and Bande 'gang' (fem, NM) may be unlikely to occur all in the same context, they illustrate the dimension of the problems of segmentation and lexical assignment, which in turn con- 
Figure 6: hypotheses for Sund

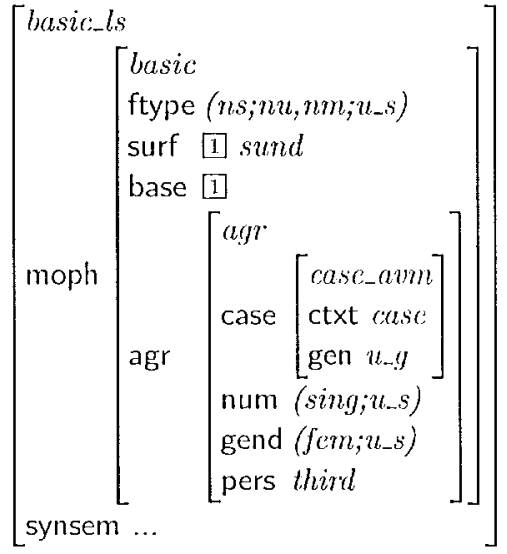

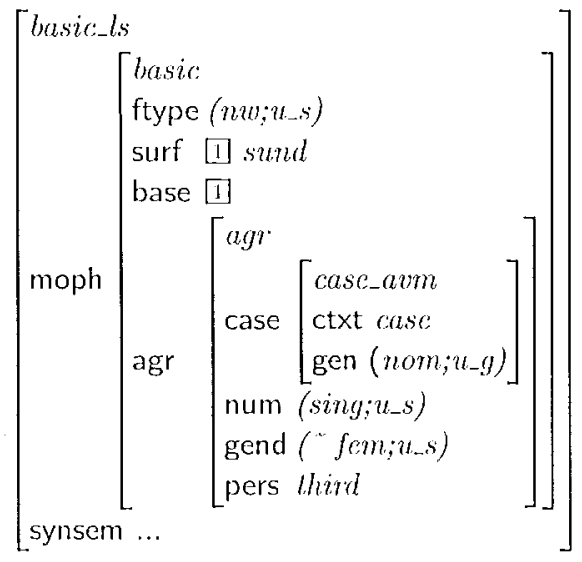

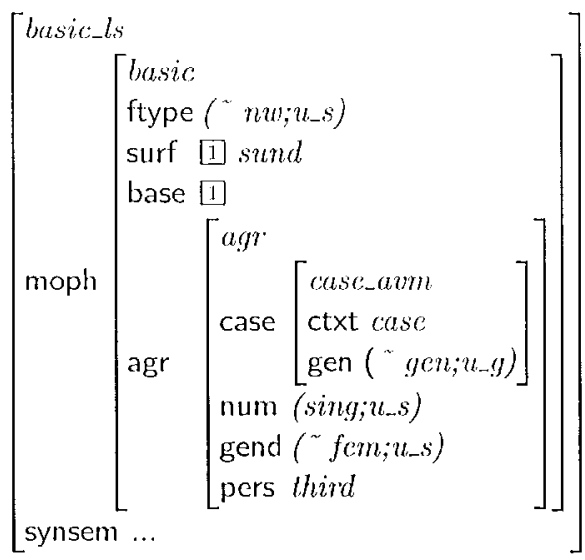

stitute part of the more general problem of disambiguation in natural language processing. We have no magic solution for the latter, but in our approach such examples must be handled with disjunctive representations until the context provides the necessary disambiguating information.

\section{An alternative model using finite-state techniques}

Alternatively, the incremental identification of inflectional types can be modelled within the framework of finite-state automata (cf. Sproat (1992)) without recourse to unification-based grammar formalisms. A FSA can be defined that has an alphiabet consisting of vectors specifying the stem shape and ending (and thus the segmentation) as well as the agreement information of possible word forms. Starting in an initial state corresponding to the constraints that apply to all unknown words, the FSA is moved by successive forms of an unknown lexeme together with their agreement information into successor states that capture the incrementally accrued inflectional information. 'The FSA may reach a final state, in which case the inflectional class has been uniquely identified, or it may remain in a nonfinal state. A lexicon would simply record the latest state reached for each noun.

Implementation of this model is greatly complicated by the problems of disambiguation just discussed in 85 . In gencral, the states of the FSA must capture disjunctions not only of inflectional classes, but also of segmentation and gender altematives. The application of automatic induction techniques to colpora appears to be essential, and we are currently pursuing possibilitics for this.

\section{Conclusion}

We have taken the inflection of German nouns to illustrate a general type-based approach to handling unknown words and the incremental accrual of their lexical information. 'The technicues can be applied not only to other classes of inflected words and to other languages, but also to other aspects of lexical information such as the valency of verlos. This may allow practical systems for natural language processing to be cuhanced so as to utilize input information that otherwise is discarded as noise.

\section{References}

Petra Barg and Markus Walther. 1998. Processing unknown words in HPSG. In Proceedings of COLING-ACL'98, pages 91-95, Montreal.

Steven Bird and Ewan Klein. 1994. Phonological analysis in typed feature systems. Computational Jinguistics, 20:455-491.

Michael R. Brent. 1991. Automatic acquisition of subcategorization frames from untagged text. In Procedings of 29th ACL, pages 209-214, Berkeley.

Lynne Cahill and Gerald Gazdar. 1999. German noun inflection. Journal of Linguistics, 35:1-42.

Robert Carpenter. 1992. The Logic of Typed Feature Structures. Cambridge University Press. 
Harald Clahsen. 1999. Lexical entries and rules of language: A multidisciplinary study of Gorman inflection. Behavioral and Brain Sciences.

Jochen Dörre and Michael Dorna. 1993. CUF a formalism for linguistic knowledge representation. In Jochen Dörre, editor, Computational Aspects of Constraint-Based Linguistic Description. IMS, Universität Stuttgart. Deliverable R1.2.A, DYANA-2 - ESPRIT Project 6852.

Karen Ehrlich and William J. Rapaport. 1997. A computational theory of vocabulary extension. In Proceedings of the 19th Annual Conference of the Cognitive Science Society, pages 205-210.

Tomaž Erjavec. 1996. Unification, Inheritance and Paradigms in the Morphology of Natural Languages. Unpublished doctoral dissertation, University of Ljubljana.

Udo Hahn, Manfred Klenner, and Klemens Schnattinger. 1996. Learning from texts - a terminological meta-reasoning perspective. In S. Wermter, E. Riloff, and G. Scheler, editors, Connectionist, Statistical, and Symbolic Approaches to Learning for Natural Language Processing, pages 453-468. Springer, Berlin.

James Kilbury, Petra Barg, and Ingrid Renz. 1994. Simulation lexikalischen Erwerbs. In Sascha Felix, Christopher Habel, and Gerd Rickheit, editors, Kognitive Linguistik: Repräsentation und Prozesse, pages 251-271. Westdoutschor Verlag, Opladen.

Jean-Pierre Kocnig. 1999. Lexical Relations. CSLI Publications.

Hans-Ulrich Kricger and John Nerbonne. 1993. Feature-based inheritance networks for computational lexicons. In Ted Briscoe et al., editor, Inheritance, Defaults, and the Lexicon, pages 90136. Cambridge University Press, Cambridge.

Detmar Meurers and Guido Minnen. 1997. A computational treatmont of lexical rules in HPSG as covariation in lexical entries. Computational Linguistics, 23:543-568.

Martin Neef. 1998. A case study in declarative morphology: German case inflection. In Wolfgang Kehrein and Richard Wiese, editors, Phonology and Morphology of the Germanic Languages, pages 219-240. Max Nicmeyer Verlag, 'Tübingen.

Carl Pollard and Ivan Sag. 1994. Head-Driven Phrase Structure Grammar. Chicago University Press.

Susanne Riehemann. 1998. 'Type-based derivational molphology. Journal of Comparative Germanic Linguistics, 2:49-77.

Richard Sproat. 1992. Morphology and Computation. MIT Press, Cambridge, Mass.

Harald Trost. 1993. Coping with derivation in a morphological component. In Proceedings of $6 \mathrm{th}$ $E A C L$, pages $368-376$.
Markus Walther and Petra Barg. 1998. 'Towards incremental lexical acquisition in HPSG. In Proceedings Joint Conference on Formal Grammar, Head-Driven Phrase Structure Grammar, and Categorial Grammar, Saarbrücken.

Dicter Wunderlich. 1999. German noun plural reconsidered. Manuscript, University of Düsseldorf. 